\title{
Otolaryngologic manifestations of HIV infection
}

\author{
B. Viswanatha, Nisha Krishna \\ Bangalore Medical College \& Research Institute, Bangalore, India
}

Correspondence: B.VISHWANATH. Address: ENT Department, Bangalore Medical College \& Research Institute, Fort, K R Road, Bangalore -560002, Karnataka, India. Telephone: 91-984-5942-832. E-mail: drbviswanatha@yahoo.co.in

Received: December 18, 2011 Accepted: May 1, $2012 \quad$ Published: June 1, 2012

DOI : $10.5430 / \mathrm{jhm} . \mathrm{v} 2 \mathrm{n} 2 \mathrm{p} 33$

URL: http://dx.doi.org/10.5430/jhm.v2n2p33

\begin{abstract}
HIV is a retrovirus on the Lentivirus subfamily. The virus binds to the CD4 receptor on the T helper lymphocytes. Fusion of the viral and cell membranes allows entry of the viral core into the cell. The reverse transcriptase enzyme, a protein carried by the virus that allows transcription of ribonucleic acid (RNA) into deoxyribonucleic acid, then mediates transcription of the viral RNA genome into viral DNA. Viral integrase then facilitates incorporation of the viral DNA into the host genome. The viral DNA is then transcribed into multiple RNA copies by the host cell. This newly created RNA is translated into viral proteins. Translation of the viral RNA sequences results in protein precursors that undergo proteolytic processing by a viral protease that liberates the functional viral proteins ${ }^{[1,2]}$.

As the virus infects and damages helper $\mathrm{T}$ cells, both humoral and cell-mediated immunity are impaired. Defective chemotaxis and phagocytosis causes increased vulnerability to infections such as candidiasis and toxoplasmosis. A lack of T-cell stimulation of B cells results in decreased immunoglobulin production and a vulnerability to encapsulated organisms such as Streptococcus pneumoniae and other bacteria. These problems are compounded by impaired neutrophil function ${ }^{[2]}$.
\end{abstract}

\section{Key words}

Otolaryngologic, HIV infection, Manifestations

\section{Otolaryngologic manifestations of HI V}

It has been showed in a number of studies that over $90 \%$ of patients with HIV develop head and neck manifestations ${ }^{[3,4]}$. In a study by Prasad et al. ${ }^{[4]}$, where 968 patients were taken up 779 presented with otolaryngologic manifestations. Of these oral and oropharyngeal lesions were one of the most common manifestations, comprising $39 \%$ of the total otolaryngologic manifestations among the AIDS patients.

\section{Oral manifestations}

Oral candidiasis is one of the earliest and most common findings suggesting HIV infection and is characterised by a typical cottage cheese inflammatory appearance. These patients usually still appear healthy, but if a known HIV-positive patient develops thrush, long-term survival appears to be diminished.

Four different types of oral candidiasis can be identified: 
- Pseudomembranous candidiasis. This is the classic form identified by a white plaque that can be scraped off, leaving an erythematous, bleeding base.

- Hyperplastic candidiasis. These lesions can easily be confused with leukoplakia or oral hairy leukoplakia since they appear as thick white plaques that cannot be scraped off. If there is any doubt regarding the diagnosis, a biopsy is indicated.

- Atrophic candidiasis. This presents as flat atrophic lesions involving the oral and oropharyngeal mucosa.

- Angular cheilitis. These are oral commissure erythematous lesions that can involve the adjacent skin.

Topical agents are usually effective in the early stages of HIV infection, but with advanced disease and increasing immune deficiency, systemic therapy (With fluconazole) may be indicated. Oesophageal or pharyngeal candidiasis is more common in this patient population and should be suspected in patients with oral candidiasis who develop a severe sore throat or difficulty in swallowing ${ }^{[3]}$.

Oral hairy leukoplakia is a condition almost pathognomonic of HIV infection and often indicates progression to AIDS.

- The lesion most frequently appears on the lateral aspect of the tongue with a thick, vertically correlated ('hairy') whitish plaque, very similar in appearance to the hyperplastic type of oral candidiasis.

- Potassium hydroxide $(\mathrm{KOH})$ preparations of surface scrapings will identify the mycelia or hyphae seen in candidiasis and can therefore be used to differentiate between these two conditions.

- A biopsy of the lesion will be diagnostic of oral hairy leukoplakia. The Epstein-Barr virus has been identified as the most likely causative agent.

- Oral hairy leukoplakia is typically asymptomatic and does not usually require any treatment, but it is a significant finding because of its diagnostic and prognostic implications. The lesions have been successfully treated with aciclovir (2g/day), sulpha drugs, zidovudine or topical retinoic acid ${ }^{[2,3]}$.

Herpes simplex infections: are more common in those infected with HIV, with herpes labialis being the most common manifestation.

- These 'fever blisters' are larger, more numerous, recur more often and persist for longer periods. Herpes simplex virus type 1 (HSV-1) is most commonly involved and usually begins as small bullae that rupture and then coalesce.

- It can extend onto the adjacent facial skin and form giant herpetic lesions. If the diagnosis is in doubt then smears or scrapings should be taken before starting treatment.

- Mild oral herpes infections can usually be treated conservatively, but high-dose oral acyclovir (Up to 4g/day) should be used for more severe lesions. Aciclovir will only be effective in eradicating the lesions if started within the first few days of infection. Prophylactic aciclovir can be used in the severely immunocompromised patient with frequent recurrences ${ }^{[2,3]}$.

Periodontal disease, gingivitis and necrotising gingivitis: are much more severe in the HIV-positive patient and can produce significant morbidity with extensive tissue destruction.

- HIV-associated gingivitis usually begins with erythematous, bleeding gums after minor trauma and can then rapidly progress to a necrotising process known as acute necrotising ulcerative gingivitis ${ }^{[3]}$.

- The mainstay of therapy for HIV-related gingivitis and periodontitis includes dental plaque removal and oral rinses with $10 \%$ povidone-iodine with $0.1 \%$ to $0.2 \%$ chlorhexidine gluconate. Management of acute necrotising 
ulcerative gingivitis and necrotizing stomatitis includes a combination of local care and systemic antianaerobic drug therapy. The necrotic tissue should be débrided, the teeth scaled, and parenteral antibiotic therapy with clindamycin or metronidazole ${ }^{[2]}$.

- Recurrent aphthous ulcers: Are frequently encountered in the HIV-positive patient and present as painful ulcers on the non-keratinised unattached oral mucosa ${ }^{[3]}$.

- $\quad$ HIV-infected patients frequently present with giant aphthous ulcerations ${ }^{[4]}$.

- Management should focus on ruling out malignancy, providing symptomatic relief, and monitoring nutritional status. The edge of the ulcer should be biopsied and submitted for histopathology to rule out lymphoma. The size, chronicity, and exquisite tenderness of major aphthous ulcers mandate close monitoring of body weight and the provision of liquid nutritional supplements. Aphthous ulcers are treated with topical corticosteroids, such as triamcinolone or flucinonide and applied up to six times per day. Other topical preparations include tetracycline (250mg per $5 \mathrm{~mL}$ ) used as a mouth wash four times/day for 4 days, or chlorhexidine $0.1 \%$ to $0.2 \%$ twice per day for 7 days. The prudent use of systemic corticosteroids may be necessary in major aphthous ulcers ${ }^{[2]}$.

Neoplastic disease in the oral cavity

Oral Kaposi's sarcoma is the most common neoplasm found in AIDS patients, and is associated with advanced immune suppression.

- Oral Kaposi's sarcoma prevalence is reported to be $1.7 \%$ to $20 \%$. Oral Kaposi's sarcoma lesions usually appear as asymptomatic reddish-purple macular or papulonodular lesions that do not blanche with compression. The palate, followed by the gingiva and tongue, are the most common locations.

- Possible treatment includes surgical removal or debulking, laser destruction, chemotherapy, either systemic or intralesional injection of vincristine or vinblastine, alone or in combination ${ }^{[5]}$.

Lymphomas: Are the second most common malignancy in HIV infection, and oral cavity involvement is possible.

- Oral lymphomas are predominantly of the extranodal non-Hodgkin's lymphoma (NHL) type and B cell lineage, and may present as rapidly growing masses in the gingiva, palate with bone destruction, tongue, or tonsillar fauces.

- Treatment involves systemic multidrug chemotherapy with methotrexate, bleomycin, doxorubicin, cyclophosphamide, vincristine, and dexamethasone, with or without radiation ${ }^{[5]}$.

Parotid gland enlargement occurs in approximately $0.8 \%$ of adults with HIV and $4 \%$ to $47 \%$ of children with HIV.

- Parotid enlargement is usually bilateral in nature, associated with cervical lymphadenopathy.

- Management of cystic enlargement of the parotid primarily involves observation. At present, effective antiretroviral therapy or oral prednisone may offer the best success in eliminating parotid enlargement. When lymphoepithelial cysts result in severe facial deformity, radiation therapy proves successful at reducing gland size and is preferred to superficial parotidectomy; however, xerogenic doses are required and radiation of benign lesions predisposes to secondary malignancies ${ }^{[5]}$.

\section{Neck manifestations}

The most common finding in the neck is cervical lymphadenopathy ${ }^{[4]}$. Apart from reactive lymphadenitis, cervical lymphadenopathy may be due to:

Infectious 
- Mycobacterial lymphadenitis: Tuberculous and atypical organisms

- Pneumocystis lymphadenitis

- Pneumocystis thyroiditis

- Viral lymphadenitis: Cytomegalovirus, Epstein-Barr virus

- Toxoplasma lymphadenitis

- Bacterial lymphadenitis or abscess secondary to

- Oropharyngeal infection

- Cat-scratch disease

Neoplastic

- Lymphoma

- Non-Hodgkin's

- Hodgkin's disease

- Metastatic Kaposi's sarcoma

- Metastatic carcinoma

- Metastatic melanoma

- Salivary gland tumours

- $\quad$ Thyroid tumours

Idiopathic

- Persistent generalized Lymphadenopathy

- Lymphoepithelial cysts of the parotid gland

Persistent generalized lymphadenopathy: Is a common early symptom of HIV infection and a common cause of cervical adenopathy.

- Persistent generalized lymphadenopathy is defined as lymphadenopathy without an identifiable infectious or neoplastic etiology, which involves two or more extrainguinal sites for at least 3 months, in a patient at risk for or confirmed to be HIV infected.

- The neck is the third most common site of Persistent generalized lymphadenopathy after the axillary and inguinal regions. Within the neck, cervical adenopathy occurs in the posterior triangle (85\%), the preauricular and postauricular regions $(51 \%, 47 \%)$, the anterior triangle (37\%), and the occipital region $(30 \%)^{[2]}$.

- FNAC (Fine needle aspiration cytology) should be the initial method of tissue sampling in most cases of suspicious cervical lymphadenopathy in HIV-infected patients ${ }^{[2,3]}$.

Three histologic patterns (A, B, and C) have been described ${ }^{[6]}$.

Pattern A, which is seen in acute HIV infection, is characterized by serpiginous and greatly enlarged lymphoid follicles with reactive germinal centers. The mantle zone around these follicles is often diminished or absent. Aggregates of small 
lymphocytes may focally penetrate into the germinal centers and contribute to their disruption (the so-called follicle lysis). Hemorrhage, monocytes, pale monocytoid B cells, and scattered multinucleated giant cells can be found between follicles.

Pattern B is seen in subchronic HIV infection. There is effacement of the follicles secondary to a progressive involution of germinal centers, depletion of lymphocytes, accumulation of plasma cells, and excessive interfollicular proliferation of blood vessels.

Pattern C, seen in late acquired immunodeficiency syndrome, is characterized by small or absent follicles with hyalinized "burnt-out" germinal centers, an almost complete depletion of lymphocytes, an increase in plasma cells, and an excessive proliferation of blood vessels.

\section{Otological manifestations}

The otological manifestations include seborrheic dermatitis of external ear, otitis externa with otomycosis, secretory otitis media, chronic otitis media, sensory neural hearing loss, and vertigo. Otitis externa is caused by Pseudomonas aeruginosa and otomycosis is often caused by Candida albicans. There has been no report of an increased incidence of these conditions in AIDS patients. Secretory otitis media secondary to nasopharyngeal lymphoid hyperplasia is common in HIV-positive patients. Sensorineural hearing loss, both unilateral and bilateral, occurs in $21 \%$ to $49 \%$ of HIV-infected patients ${ }^{[7]}$. Williams ${ }^{[8]}$ in his study found otological manifestations of HIV in $80 \%$ of patients. In the series by Prasad et al. ${ }^{[4]}$ of 968 patients, otological manifestations were seen in $20 \%$ of patients.

\section{Nasal and paranasal sinus problems associated with HIV}

Several studies of patients infected with HIV have described a $30 \%$ to $68 \%$ prevalence of sinusitis ${ }^{[8]}$. The bacteriology of acute sinusitis in this population includes the same organisms normally considered in the immunocompetent patient. In late stages of HIV infection, atypical opportunistic fungal sinusitis with Alternaria alternata, Aspergillus, Pseudallescheria boydii, Cryptococcus, and Candida albicans can occur. Clinicians must rigorously use a dual approach, which includes an appropriate antibiotic as well as a decongestant. Oral antibiotic therapy should continue for a minimum of 3 weeks. In patients resistant to therapy, intravenous antibiotics, surgical drainage, or both are imperative.

\section{Otolaryngologic manifestations in children with HIV}

The majority of children are infected vertically, i.e. infection of the infant from an infected mother in the pre-, peri-, or post-natal periods. The most common presenting features include persistent generalised lymphadenopathy, hepatosplenomegaly, chronic/recurrent diarrhoea, poor growth, and fever. Fifteen to twenty percent of untreated children will present with an AIDS-defining illness by 12 months. mastoiditis, tonsillitis, orbital/peri-orbital cellulitis, oral candidiasis, and dental infections. Infections with streptococcus pneumoniae and group A streptococcus are common, and often progress to severe systemic infection with an appreciable mortality. Anti-retroviral medicines given to the mother and then to the baby, both before and after delivery, have played an important part in the reduction of the transmission rate. There are various combinations of nucleoside and non-nucleoside reverse transcriptase inhibitors that are commonly used for children. By and large, the protease inhibitors tend to be avoided as first line therapy because of attendant side effects $^{[9]}$.

During recent years, from all geographic areas a progressive increase of mean age at diagnosis of HIV infection and AIDS has been recorded ${ }^{[10]}$. 


\section{Conclusion}

Awareness of the opportunistic infections in HIV infections, is inevitable for early diagnosis and treatment of the same in view of the fact that instillation of HAART (Highly active antiretroviral therapy) in the initial stages of the disease significantly helps in prolonging and improving the quality of life in the patients.

\section{Conflict of interest}

The author declares that there is no conflict of interest statement.

\section{References}

[1] USAID, INDIA; HIV/AIDS Health Profile, December 2010.

[2] Pletcher SD, Goldberg AN. Head and neck manifestations of human immunodeficiency virus infection. Cummings: Otolaryngology: Head \& Neck Surgery, 4th Ed. 2005 Mosby, Inc.

[3] Lubbe DE, HIV and ENT. CME 2004; 22: 250-53

[4] Prasad KC, Bhojwani KM, Shenoy V, Prasad SC, HIV manifestations in otolaryngology. American Journal of Otolaryngology-Head and Neck Medicine and Surgery 2006; 27:179-85.

[5] Patton LL, Horst CV. Oral Infections and Other manifestations of HIV Disease. Infectious disease clinics of North America1999; 13:879-900. http://dx.doi.org/10.1016/S0891-5520(05)70114-8

[6] Caponetti G, Pantanowitz L, HIV-associated lymphadenopathy. Ear Nose Throat J 2008;87(7):374-75. PMid: 18633927.

[7] Lalwani AK, Sooy CD. Otologic and neurotologic manifestations of acquired immunodeficiency syndrome. Otolaryngol Clin North Am 1992;25:1183-97.

[8] Williams MA. Head and neck findings in pediatric acquired immune deficiency syndrome. Laryngoscope 1987;97:713-16. PMid: 3586813. http://dx.doi.org/10.1288/00005537-198706000-00013

[9] Hoare S. HIV infection in children-impact upon ENT Doctors. International Journal of Pediatric Otorhinolaryngology 2003;67S1:S85-S90

[10] Manfredi R. HIV infection and advanced age Emerging epidemiological, clinical, and management issues. Ageing Research Reviews 2004;3:31-54. PMid: 15164725. http://dx.doi.org/10.1016/j.arr.2003.07.001 